J3eA, Journal sur l'enseignement des sciences et technologies de l'information et des systèmes, Volume 4, Hors-Série 2, 15 (2005)

DOI : http://dx.doi.org/10.1051/bib-j3ea:2005715

(C) EDP Sciences, 2005

Mise en oeuvre d'une technique de régularisation pour l'estimation de paramètres physiques : application à un banc d'essai électro-mécanique

P. Sibille ${ }^{1}$ et C. Defranoux-Igier ${ }^{2}$

${ }^{1}$ Centre de Recherche en Automatique de Nancy

CNRS UMR 7039

Université Henri Poincaré, Nancy 1

BP 239

F-54506 Vandoeuvre CEDEX, France

${ }^{2}$ Laboratoire d'Informatique, Signaux et Systèmes de Sophia Antipolis

UMR 6070 UNSA CNRS

200 route des lucioles

Les Algorithmes, Bâtiment Euclide B, BP 121

F-06903 Sophia-Antipolis CEDEX, France 


\title{
MISE EN CEUVRE D'UNE TECHNIQUE DE RÉGULARISATION POUR L'ESTIMATION DE PARAMẼTRES PHYSIQUES : APPLICATION À UN BANC D'ESSAI ÉLECTRO-MÉCANIQUE
}

\author{
Patrick Sibille $^{1}$, Céline Defranoux-Igier ${ }^{2 \dagger}$ \\ ${ }^{1}$ Centre de Recherche en Automatique de Nancy, CNRS UMR 7039 \\ Université Henri Poincaré, Nancy 1, BP 239, 54506 Vandouvre Cedex, France. \\ E-mail :Patrick.Sibille@cran.uhp-nancy.fr \\ ${ }^{2}$ Laboratoire d'Informatique, Signaux et Systèmes de Sophia Antipolis, UMR 6070 \\ UNSA CNRS, 2000 route des lucioles, Les Algorithmes - Bât. Euclide B, BP 121, \\ 06903 Sophia Antipolis Cedex, France.
}

\begin{abstract}
Résumé : Cette manipulation a pour objectif de mettre en œuvre une technique de régularisation par critère composite pour estimer directement les paramètres physiques d'un modèle à temps continu représentant un banc électro-mécanique simple et classique. Sur cette application, nous illustrons la notion d'identifiabilité locale et les problèmes classiques liés aux méthodes locales d'optimisation (choix des paramètres initiaux, conditionnement du hessien du critère, estimation d'incertitudes paramétriques,...). Il est également montré comment l'introduction de connaissances paramétriques peut pallier le problème d'identifiabilité du modèle et mener ainsi à la seule solution physiquement acceptable. Une exploitation originale des expressions analytiques des critères régularisé et non régularisé en comparaison avec les estimations obtenues permet une validation de la structure du modèle simplifié.
\end{abstract}

Mots clès : Paramètre physique, estimation paramétrique, optimisation, régularisation, pénalité, connaissance $a$ priori, système électro-mécanique.

\section{INTRODUCTION}

Dans le cadre du module d'enseignement, consacré à l'identification, les étudiants d'une filière de type DESS ou ingénieurs, perçoivent souvent de nombreuses notions de ce cours comme mathématiques, abstraites et peu exploitables dans un contexte applicatif. La manipulation, présentée ici, est destinée aux étudiants de DESS spécialisés dans le domaine de l'électrotechnique. Elle tire profit d'un banc de machines à courant continu et a pour objet d'illustrer quelques points de ce cours dédié, en partie, à l'《identification de modèles boîte grise » [1].

Dans sa démarche, l'automaticien exploite la plupart du temps un modèle qui reproduit aussi fidèlement que possible le comportement du système qu'il cherche, par exemple, à commander. Pour ce faire, il procède à la phase usuellement dénommée identification qui s'appuie sur des lois statistiques

†Actuellement à l'École Supérieure d'Ingénieurs en Électronique et Électrotechnique, Antenne de Sophia-Antipolis, rue Albert Einstein, BP 085, 06902 Sophia-Antipolis, Email : igierc@esiee.fr pour aboutir à un modèle qualifié de boîte noire ou de représentation [2,3]. Cependant, dans un grand nombre de domaines applicatifs comme la robotique $([4,5,6])$, la mécanique $([7,8])$, l'électrotechnique $([9,10,11,12,13])$ et l'automobile $([14,15,16])$, il ne suffit pas de disposer d'un modèle falsifiant de façon satisfaisante les données expérimentales, il faut en plus que celui-ci ait des paramètres qui puissent avoir un sens pour le spécialiste du domaine d'application. Pour obtenir de tels modèles dits de connaissance ou boîte blanche, les lois de la physique sont classiquement exploitées. On parle alors de modélisation physique.

Les deux approches précédentes peuvent revêtir des difficultés liées à la complexité des modèles obtenus ou a contrario à leur trop grande simplicité. Une alternative à ces deux méthodologies extrêmes est apparue dans les années 90 : il s'agit de l'approche qualifiée de boîte grise. La méthodologie enseignée, ici, s'inscrit dans ce cadre. Elle repose sur des techniques d'optimisation classique par programmation non linéaire [17]. Pour remédier aux problèmes qui en découlent - identifiabilité structurelle [18] et choix du minimum physiquement ad- 
missible parmi l'ensemble des minima locaux et/ou globaux - des connaissances a priori sont prises en compte sous la forme de termes de pénalité extérieure ou contrainte, dans une procédure d'optimisation itérative de façon à obtenir une estimation des paramètres physiquement admissible, c'està-dire dont les intervalles d'incertitude sont compatibles avec les connaissances a priori.

Le premier objectif de cette manipulation est de montrer que l'automatique - pour estimer les paramètres d'une machine - peut proposer une alternative intéressante aux techniques spécifiques classiques menées en électrotechnique. Ainsi pour ces étudiants, c'est à nouveau l'occasion d'évaluer les bancs qui ont servi dans le module « machines électriques », mais cette fois-ci avec un éclairage pédagogique différent qui met en œuvre les outils d'optimisation en intégrant tout ou partie des savoirs préalablement acquis. Le deuxième objectif vise à mettre en lumière l'intérêt d'exploiter des modèles issus de la physique (lorsque c'est possible) de façon à introduire facilement de la connaissance paramétrique a priori. Le troisième objectif ambitionne de montrer qu'il ne suffit pas toujours de disposer d'un modèle «physique » reproduisant correctement les grandeurs mesurées, pour que celui-ci conduise à des paramètres qui recèlent eux aussi un sens physique. En outre, ce banc didactique est très attrayant puisqu'il permet, par le choix de conditions expérimentales adéquates, d'illustrer les notions d'identifiabilité structurelle et de minima locaux et globaux.

Dans cet article, la démarche enseignée - fondée sur la minimisation d'un critère composite, constitué d'une partie relative à la fidélité aux données et d'une autre reflétant les informations a priori est mise en œuvre de façon très satisfaisante sur un banc électro-mécanique expérimental. Ce système est formé d'un moteur couplé à une génératrice. Il est exploité de façon didactique pour illustrer les avantages de cette méthodologie. La procédure étudiée assure l'estimation directe du vecteur de paramètres physiques de ce banc en fonctionnement normal et fournit une estimation de la matrice de covariance de ces paramètres. A travers l'exploitation des résultats expérimentaux, il est montré aux étudiants :

- que l'approche présentée permet de pallier le problème d'identifiabilité locale du modèle considéré, - qu'il ne suffit pas d'introduire, au niveau de la phase d'initialisation d'un algorithme d'optimisation, les connaissances a priori pour converger vers une solution physiquement admissible,

- que compte tenu des hypothèses posées, les expressions analytiques des critères régularisé et non régularisé sont exploitées pour prouver, dans notre cas, que l'introduction du terme de pénalité assure l'unicité de la solution recherchée,

- enfin, que les manipulations de ces mêmes expres- sions permettent d'illustrer la cohérence entre les résultats expérimentaux et ceux qui découlent de l'étude théorique. La validité du modèle simplifié choisi est notamment confortée par cette cohésion.

Le travail relatif à la mise en œuvre de cette méthodologie comporte 4 parties principales. Dans la première partie, les étudiants posent leurs hypothèses et effectuent la modélisation. Le protocole expérimental est ensuite justifié et mis en œuvre sur le système électrotechnique. Puis, les campagnes de mesures sont analysées et pré-traitées. Le travail suivant est consacré à la comparaison des résultats théoriques et expérimentaux. Enfin, les étudiants formulent leurs conclusions. Le plan de ce papier suit le travail demandé.

\section{POSITION DU PROBLĖME}

Nous nous intéressons à des systèmes multivariables pouvant être représentés par des modèles à temps continu décrits par un modèle d'état. Lors des simulations, le modèle d'état continu est considéré sous sa forme discrète puisque seules les mesures échantillonnées $u_{k}$ et $y_{k}$ des entrées-sorties continues $u(t)$ et $y(t)$ sont disponibles. Il est, également, supposé que le vecteur de sortie mesuré $y_{k} \in \mathbb{R}^{m}$ est entaché d'un bruit additif $e_{k}$ indépendant du vecteur d'entrée $u_{k}$, à moyenne nulle et de variance $\Lambda$ tel que $y_{k}=C(\theta) x_{k}+D(\theta) u_{k}+e_{k}$ où $x_{k} \in \mathbb{R}^{n}$ est le vecteur d'état non mesuré.

Le critère quadratique $J(\theta)$ à minimiser qui se fonde alors sur l'erreur de sortie est défini par :

$$
J(\theta)=\frac{1}{2} \sum_{k=1}^{N}\left(y_{k}-\hat{y}_{k}(\theta)\right)^{T} \operatorname{diag}\left(\Lambda^{-1}\right)\left(y_{k}-\hat{y}_{k}(\theta)\right)
$$

avec $N$ nombre d'observations. L'expression diag signifie que la diagonale de la matrice est considérée : $\operatorname{diag}\left(\Lambda^{-1}\right)=\left[\frac{1}{\sigma_{e_{1}}^{2}} \cdots \frac{1}{\sigma_{e_{m}}^{2}}\right]$. De nombreux algorithmes d'optimisation existent dans la littérature [17]. Toutefois, le critère (1) possède généralement plusieurs minima locaux et/ou globaux et la procédure d'optimisation ne permet pas d'assurer la convergence de l'algorithme vers le minimum global associé aux paramètres physiques. Le problème consiste alors à rechercher parmi ces solutions celle qui est physiquement acceptable. Cette recherche est facilitée en intégrant directement, au sein de la phase d'estimation, l'information a priori disponible sur certains paramètres. Une technique dite de régularisation a été ici choisie.

\section{Hypothèses de travail :}

- le système appartient à la classe de modèle,

- le modèle de connaissance peut se mettre sous forme d'état et est connu, 
- on dispose d'un lot de $N$ mesures échantillonnées de $u(t)$ et de $y(t)$ et l'état $x(t)$ n'est pas mesuré,

- les bruits sont modélisables par des variables aléatoires indépendantes, de moyennes nulles, distribuées de façon identique dont la matrice de covariance est connue,

- les fonctions et coefficients de régularisation sont choisies.

\section{Résultats attendus :}

- estimer le vecteur de paramètres physiques $\theta$,

- estimer la matrice de covariance de l'estimateur.

\section{TECHNIQUE DE RÉGULARISATION}

La théorie de la régularisation est apparue avec la résolution des problèmes inverses [19]. D'une manière générale, cette approche consiste à prendre en compte des connaissances sur le système pour améliorer la qualité du modèle [19,20]. Augmenter la quantité d'informations a priori rend alors le problème d'identification mieux conditionné, puisque plus de contraintes sont imposées sur le modèle.

La technique consiste alors à modifier le critère précédent $J(\theta)$ en ajoutant une fonction régularisante $f(\theta)$ représentant les connaissances sur les différents paramètres à intégrer, pondérée par un vecteur de coefficients $\alpha$ tel que :

$$
J_{\text {reg }}(\theta)=J(\theta)+\alpha^{T} f(\theta)
$$

Les choix de $f(\theta)$ et $\alpha$ ne sont pas discutés ici, mais restent encore des problèmes ouverts [20]. Plusieurs fonctions sont envisageables, nous en avons retenu une quadratique qui est définie pour chaque composante $\theta_{j}$ par [1] :

$$
\begin{aligned}
f_{j}\left(\theta_{j}\right) & =\left(\theta_{j}-\theta_{j, \max }\right)^{2} \mathbb{I}_{\left\{\theta_{j, \max }<\theta_{j}\right\}} \\
& +\left(\theta_{j, \text { min }}-\theta_{j}\right)^{2} \mathbb{I}_{\left\{\theta_{j}<\theta_{j, \text { min }}\right\}}
\end{aligned}
$$

avec $j=1, \ldots, n_{r}$ où $n_{r}$ correspond au nombre de paramètres sur lesquels de la connaissance est introduite et aux $n_{r}$ premières composantes de $\theta$; si ce n'est pas le cas, il est toujours possible de réordonner les paramètres. $\theta_{j, \text { min }}$ et $\theta_{j, \max }$ correspondent respectivement à la valeur de la borne inférieure et de la borne supérieure de l'intervalle décrivant la connaissance sur le paramètre $\theta_{j}$, et $\mathbb{I}_{\{x\}}$ est la fonction indicatrice de l'événement $x$ telle que : si $x$ est vérifié alors $\mathbb{I}_{\{x\}}=1$ sinon $\mathbb{I}_{\{x\}}=0$.

La solution $\hat{\theta}$ qui minimise le critère (2) est un compromis entre fidélité du modèle aux données (minimisant $J(\theta)$ ) et respect des propriétés qui reflètent la connaissance a priori (vérifiant $f(\theta)=0$ ) pondérée par un indice $\alpha$.

\section{APPLICATION}

Nous nous proposons d'appliquer la méthodologie de régularisation à des données expérimentales issues d'un banc électro-mécanique. Le dispositif expérimental est constitué d'un moteur relié à une génératrice par un accouplement rigide (Cf. figure (1)). L'entrée et la sortie sont respectivement la tension d'alimentation et la vitesse de rotation du moteur.

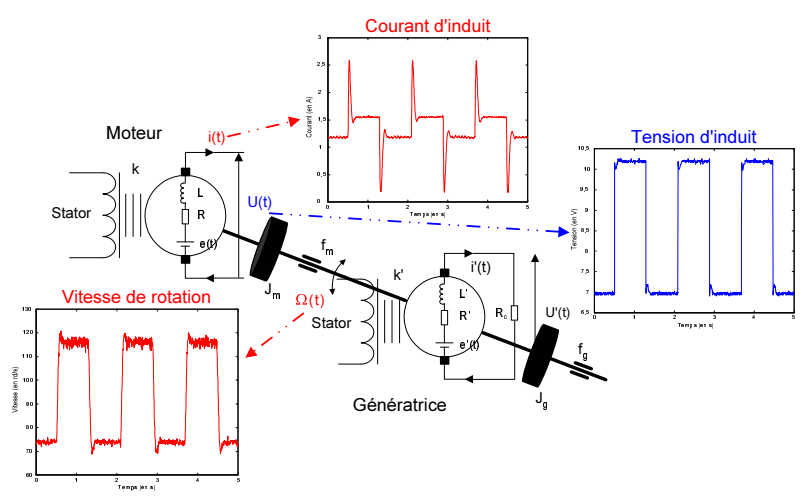

Fig. 1. Schéma de l'ensemble moteur-génératrice

Afin d'illustrer, de manière didactique, les effets de la régularisation sur ce dispositif expérimental nous poserons quelques hypothèses simplificatrices réalistes. De plus, de façon à pouvoir imager nos propos dans un espace représentable graphiquement, l'ensemble des paramètres à estimer a été limité à 3 .

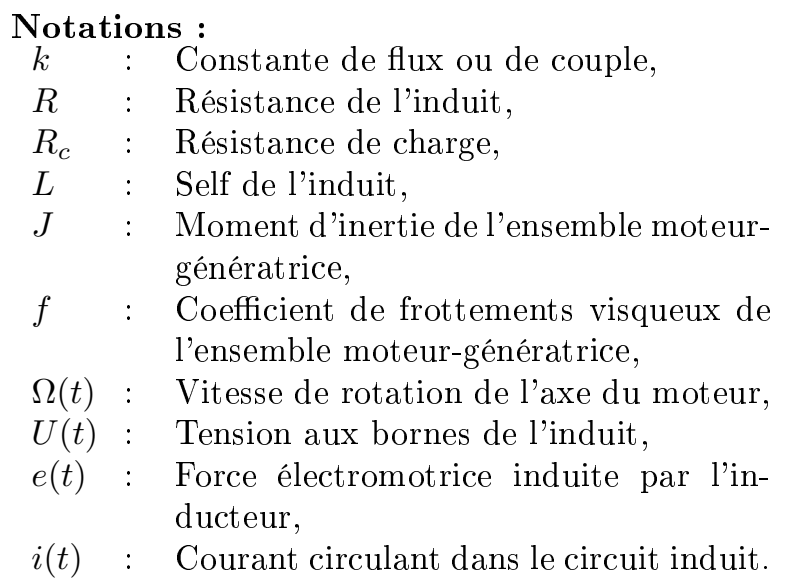

Hypothèses :

- les 2 moteurs sont supposés identiques,

- l'accouplement de ces 2 moteurs est considéré comme étant rigide,

- les frottements secs sont présumés négligeables,

- les constantes de flux et de couple sont identiques : $k_{e}=k_{t}$,

- le vecteur des paramètres physiques à estimer est : $\theta=\left[\begin{array}{lll}J & L & f\end{array}\right]^{T}$.

Ce système peut être décrit par le modèle d'état avec : 


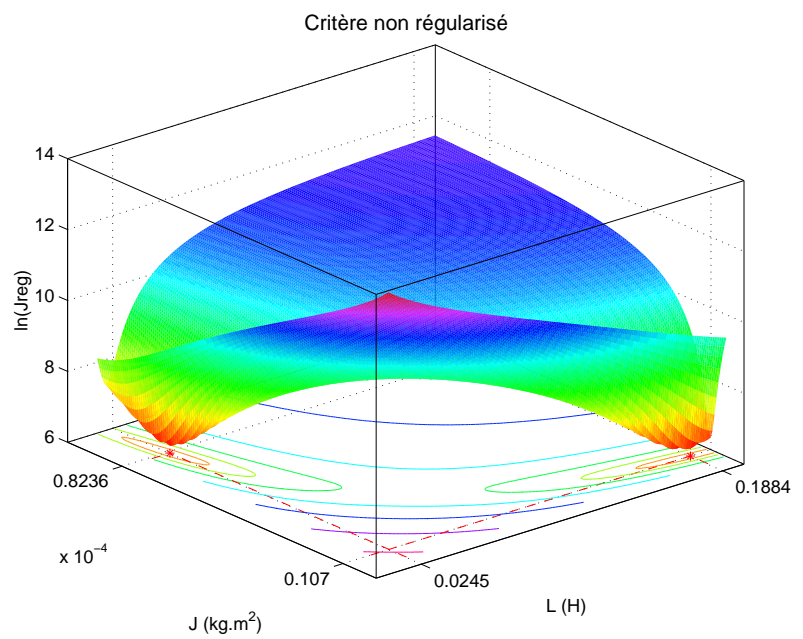

Fig. 2. Évolution du critère non régularisé dans l'espace paramétrique

$$
\begin{array}{ll}
A(\theta)=\left[\begin{array}{lc}
\frac{R}{L} & -\frac{k}{L} \\
\frac{k}{J} & -\frac{k^{2}+f\left(R+R_{c}\right)}{\left(R+R_{c}\right) J}
\end{array}\right] & B(\theta)=\left[\begin{array}{l}
\frac{1}{L} \\
0
\end{array}\right] \\
C(\theta)=\left[\begin{array}{ll}
0 & 1
\end{array}\right] & D(\theta)=\left[\begin{array}{l}
0
\end{array}\right]
\end{array}
$$

La fonction de transfert associée à ce modèle d'état s'écrit :

$$
F(s)=\frac{\Omega(s)}{U(s)}=\frac{\frac{k}{L J}}{s^{2}+p_{1} s+p_{0}}
$$

avec

$$
\begin{aligned}
& p_{1}=\frac{k^{2}}{\left(R+R_{c}\right) J}+\frac{R^{2}+R R_{c}}{\left(R+R_{c}\right) L}+\frac{f}{J} \\
& p_{0}=\frac{\left(2 R+R_{c}\right) k^{2}}{\left(R+R_{c}\right) L J}+\frac{\left(R^{2}+R R_{c}\right) f}{\left(R+R_{c}\right) L J}
\end{aligned}
$$

Sous ces hypothèses, ce modèle est localement identifiable, le critère d'erreur de sortie admet 2 minima globaux (Cf. figure (2)) donnés par :

$$
\begin{aligned}
& \theta_{1}=\left[\begin{array}{lll}
J^{*} & L^{*} & f^{*}
\end{array}\right]^{T} \\
& \theta_{2}=\left[\begin{array}{lll}
\frac{L^{*}\left(k^{2}+f^{*}\left(R+R_{c}\right)\right)}{R\left(R+R_{c}\right)} & \frac{J^{*} R\left(R+R_{c}\right)}{k^{2}+f^{*}\left(R+R_{c}\right)} & f^{*}
\end{array}\right]^{T}
\end{aligned}
$$

où, $J^{*}, L^{*}$ et $f^{*}$ représentent les valeurs recherchées.

\subsection{Définition du protocole expérimental}

Des campagnes de mesures ont été menées sur le banc expérimental. Lors de celles-ci, Des excitations sur la tension d'alimentation du moteur ont été appliquées et la vitesse de rotation de ce dernier a été mesurée (Cf. figure (1)). Les signaux sont échantillonnés avec une période de $5 \mathrm{~ms}$.

Plusieurs fichiers de mesures ont été recueillis dans des conditions expérimentales similaires. Ces campagnes de mesures ont été scindées en deux lots. Le premier a été exploité pour la phase d'identification

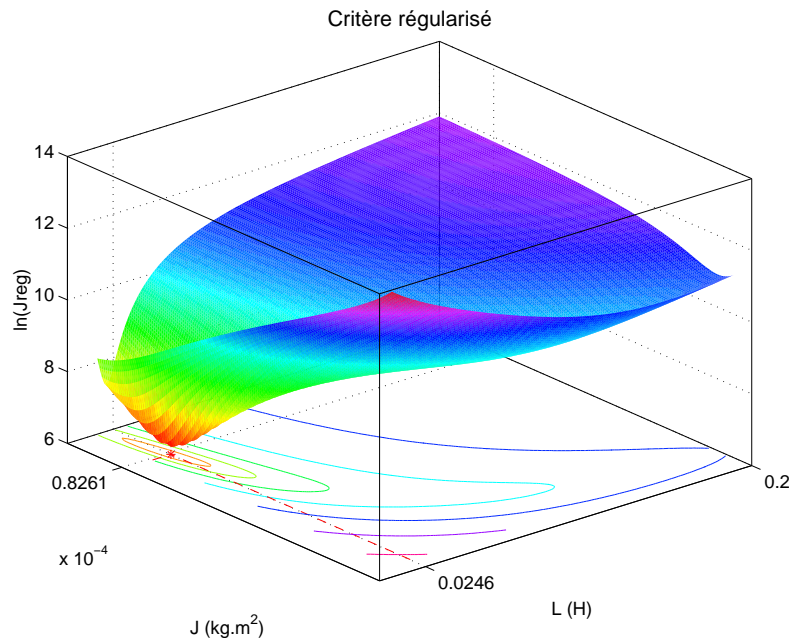

Fig. 3. Évolution du critère régularisé dans l'espace paramétrique

et le second pour la phase de validation.

Lors de l'étape d'identification seules la tension d'alimentation et la vitesse de rotation du moteur sont exploitées. En revanche pour la phase de validation, les deux grandeurs précédentes sont mesurées ainsi que le courant d'induit circulant dans le moteur. Lors de l'exploitation des données expérimentales, les connaissances introduites sont : les valeurs de la constante de flux $(k=0.063 N . m / A)$, des résistances de l'induit $(R=1.25 \Omega)$ et de charge $\left(R_{c}=\right.$ $10.3 \Omega$ ). Pour ces trois paramètres les valeurs connues a priori sont admises : elles ne seront pas ré-estimées lors de la phase d'identification. De plus, on dispose d'une connaissance de type «intervalle» sur la self de l'induit : les valeurs physiquement admissibles pour cette grandeur sont comprises entre 10 et $30 \mathrm{mH}$. La valeur initiale du vecteur des paramètres physiques est choisi égal à : $\theta_{\text {init }}=\left[\begin{array}{lll}J_{\text {init }} & L_{\text {init }} & f_{\text {init }}\end{array}\right]^{T}=$ $\left[\begin{array}{lll}1 e-5 & 25 e-3 & 5 e-4\end{array}\right]^{T}$ pour respecter les informations a priori.

\subsection{Résultats et analyse}

Dans le tableau (1) sont reportées les estimations des paramètres obtenues par la minimisation d'un critère fondé sur l'erreur de sortie (noté $\hat{\theta})$ ou par la minimisation du critère régularisé (noté $\hat{\theta}_{\text {reg }}$ ). Sur ces deux estimations du vecteur $\theta$ des paramètres, une seule est conforme aux connaissances a priori. Dans ce tableau, sont également consignés les estimations des écartstypes pour chacun des paramètres. Les optima estimés $\quad\left(\hat{\theta}_{\text {reg }}=\left[\begin{array}{lll}8.25 e-5 & 24.5 e-3 & 2.04 e-4\end{array}\right]^{T}\right.$ et $\hat{\theta}=\left[\begin{array}{lll}1.07 e-5 & 188 e-3 & 2.04 e-4\end{array}\right]^{T}$ ) satisfont bien à l'équation (7) liée à l'identifiabilité.

Remarque : les valeurs du coefficient de frottements visqueux étant identiques pour les 2 optima, il sera fixé à $f^{*}=2.04 e-4 N$.m.s $/ r d$, lors des représentations graphiques. 
De plus, en établissant l'expression analytique de l'espérance du critère d'erreur de sortie (Cf. équations (8) à (10)) en fonction du vecteur $\theta_{1}$, son évolution peut-être tracée dans l'espace paramétrique (Cf. figure (2)). Les valeurs des paramètres ainsi obtenues, qui correspondent aux optima de ce critère $\left(\left[\begin{array}{lll}8.24 e-5 & 24.5 e-3 & 2.04 e-4\end{array}\right]^{T}\right.$ et $[1.07 e-5 \quad 188 e-3 \quad 2.04 e-4]^{T}$ ), sont comparables aux précédentes : l'ensemble de ces estimations est totalement cohérente. Cette cohérence prouve que le modèle physique simplifié est bien adapté aux données expérimentales.

$$
\mathbb{E}(J(\theta))=\frac{N \sigma_{u}^{2}}{4 \sigma_{e}^{2}}\left(t_{1}+t_{2}\right)
$$

avec,

$$
\begin{aligned}
& t_{1}=\frac{\left(\delta^{2} a_{2}+\left(a_{1}^{*}\right)^{2} J L+\delta a_{1} a_{1}^{*}-L^{*} J^{*} a_{1}^{2}\right) b_{0}^{2}}{\left(\delta^{2} a_{2}+a_{1}^{* 2} J L+(\delta+2 J L) a_{1} a_{1}^{*}+L^{*} J^{*} a_{1}^{2}\right) a_{1}} \\
& t_{2}=\frac{\left(\delta^{2} a_{2}-\left(a_{1}^{*}\right)^{2} J L-\delta a_{1} a_{1}^{*}+L^{*} J^{*} a_{1}^{2}\right) b_{0}^{2}}{\left(\delta^{2} a_{2}+a_{1}^{* 2} J L+(\delta+2 J L) a_{1} a_{1}^{*}+L^{*} J^{*} a_{1}^{2}\right) a_{1}^{*}} \\
& \delta=J^{*} L^{*}-J L
\end{aligned}
$$

où, $N$ correspond au nombre d'observations. $\sigma_{u}^{2}$ et $\sigma_{e}^{2}$ représentent les variances du signal d'entrée et de la perturbation considérée comme une suite de variables aléatoires indépendantes, de moyennes nulles, distribuées de façon identique.

Sur les données d'identification, l'algorithme d'optimisation a abouti à des solutions convenables du point de vue de la minimisation des critères d'erreur de sortie simple et régularisé. Les courbes de vitesse (Cf. figures (4) et (5)) obtenues, lors de la phase de validation, attestent que les estimations des paramètres sont tout à fait satisfaisantes vis à vis d'un critère de simulation.

La simulation des deux modèles réalisée, à partir de $\hat{\theta}$ et $\hat{\theta}_{\text {reg }}$, révèle que le modèle obtenu sans régularisation ne permet pas de reconstruire correctement le courant dans l'induit, par opposition à celui qui exploite $\hat{\theta}_{\text {reg }}($ Cf. figures (6), (7) et (8)). Les mesures de courant ont été effectuées dans des conditions expérimentales similaires à celles qui ont garanti l'identification. Ces résultats corroborent les conclusions ci-avants.

\begin{tabular}{lrrr}
\hline & \multicolumn{1}{c}{$\hat{J}$} & \multicolumn{1}{c}{$\hat{L}$} & \multicolumn{1}{c}{$\hat{f}$} \\
\hline \hline$\theta_{\text {init }}$ & $1 e-5$ & $25 e-3$ & $5 e-4$ \\
$\hat{\theta}$ & $1.07 e-5$ & $188 e-3$ & $2.04 e-4$ \\
$\pm \sigma_{\hat{\theta}}$ & $\pm 0.05 e-5$ & $\pm 4.5 e-3$ & $\pm 0.07 e-4$ \\
$\hat{\theta}_{\text {reg }}$ & $8.25 e-5$ & $24.5 e-3$ & $2.04 e-4$ \\
$\pm \sigma_{\hat{\theta}_{\text {reg }}}$ & $\pm 0.14 e-5$ & $\pm 0.8 e-3$ & $\pm 0.07 e-4$ \\
\hline
\end{tabular}

Tab. 1. Estimation des paramètres et de leur écart-type avec et sans régularisation

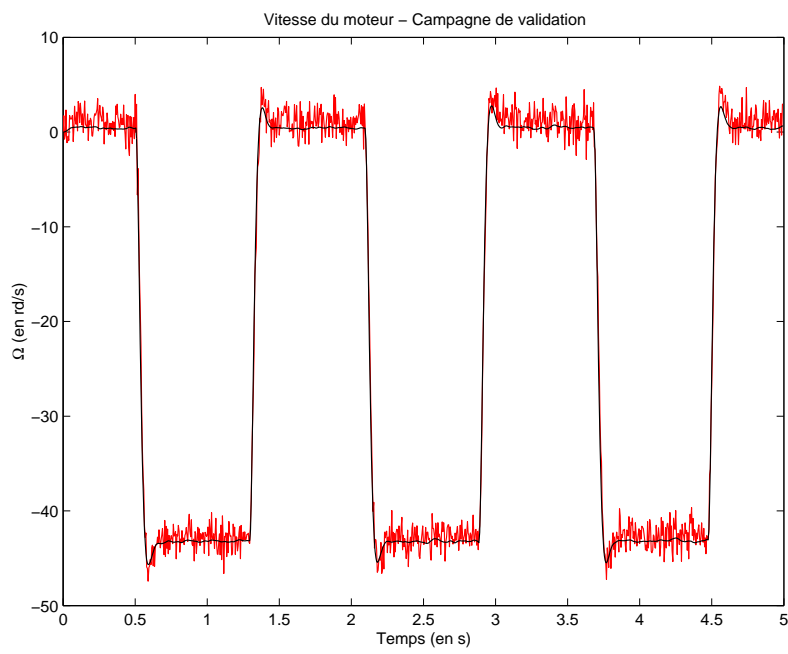

Fig. 4. Sans régularisation : évolution temporelle de la vitesse mesurée (en rouge) et simulée (en noire) du moteur

Par ailleurs, le tracé du critère régularisé (intégrant les connaissances sur la valeur de $L$ ) dans l'espace paramétrique ((Cf. figure (3)) confirme qu'il n'y a plus qu'un seul optimum correspondant aux valeurs physiques cherchées $\left(\left[\begin{array}{lll}8.26 e-5 & 24.6 e-3 & 2.04 e-4\end{array}\right]^{T}\right)$.

Les résultats obtenus, sur ce banc électromécanique, prouvent l'intérêt de la minimisation d'un critère intégrant les connaissances a priori. Ils valident les hypothèses simplificatrices faites a priori comme celles relatives aux frottements secs ou encore l'identité des caractéristiques du moteur et de la génératrice.

La figure (8) indique que les paramètres physiques estimés à partir des campagnes de mesures sont en adéquation avec les mesures complémentaires du courant circulant dans l'induit du moteur. Cette concordance révèle que pour ce type de conditions expérimentales le modèle physique simplifié falsifie, de façon satisfaisante, les données expérimentales. 


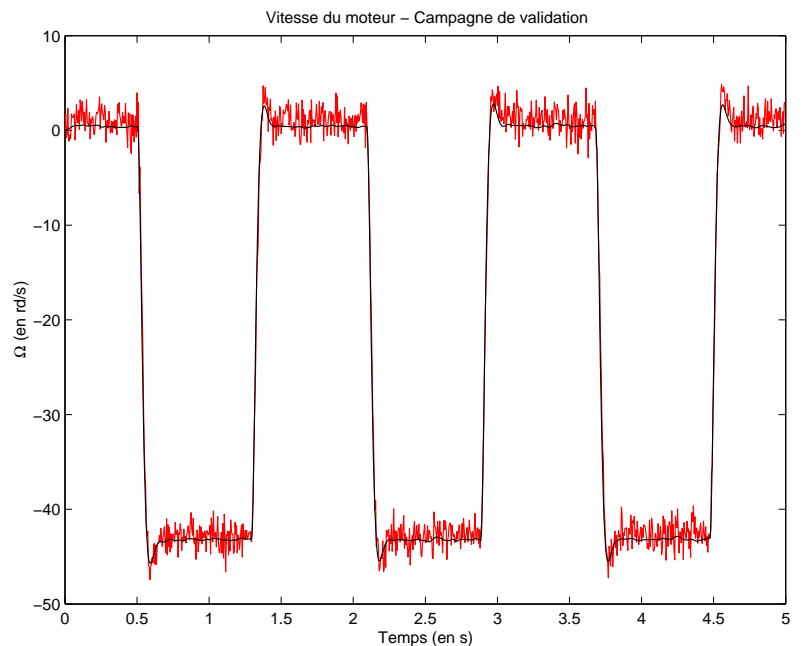

Fig. 5. Avec régularisation : évolution temporelle de la vitesse mesurée (en rouge) et simulée (en noire) du moteur

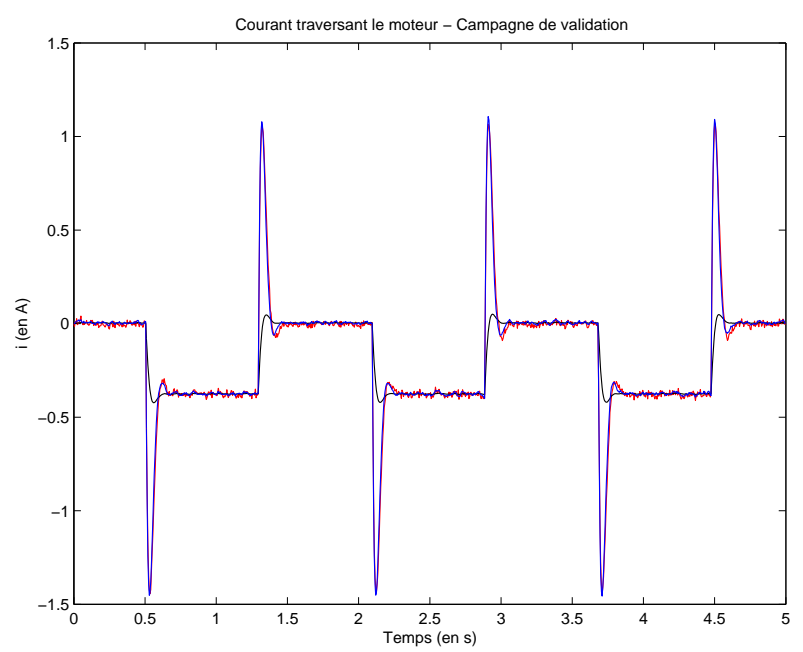

Fig. 6. Évolution temporelle des courants d'induit mesuré (en rouge) et simulés (en noir : «sans régularisation», en bleu : «avec régularisation»). Ces 2 derniers sont simulés à partir des modèles obtenus en minimisant respectivement les critères non régularisé (1) et régularisé (2)

\section{CONCLUSION}

Cette manipulation est l'occasion pour les étudiants de confronter la méthodologie de caractérisation des machines électriques des électro-techniciens et celle d'identification des automaticiens. Plutôt que d'opposer ces 2 démarches, l'approche bô̂te grise leur montre, notamment, comment tirer profit des connaissances a priori de l'électrotechnique pour améliorer la connaissance physique et converger vers une solution physiquement admissible. La méthodologie présentée est fondée sur la minimisation d'un critère composite formé d'un critère standard d'erreur de sortie auquel un terme de pénalité pondéré est ajouté. Ce terme autorise l'introduction des

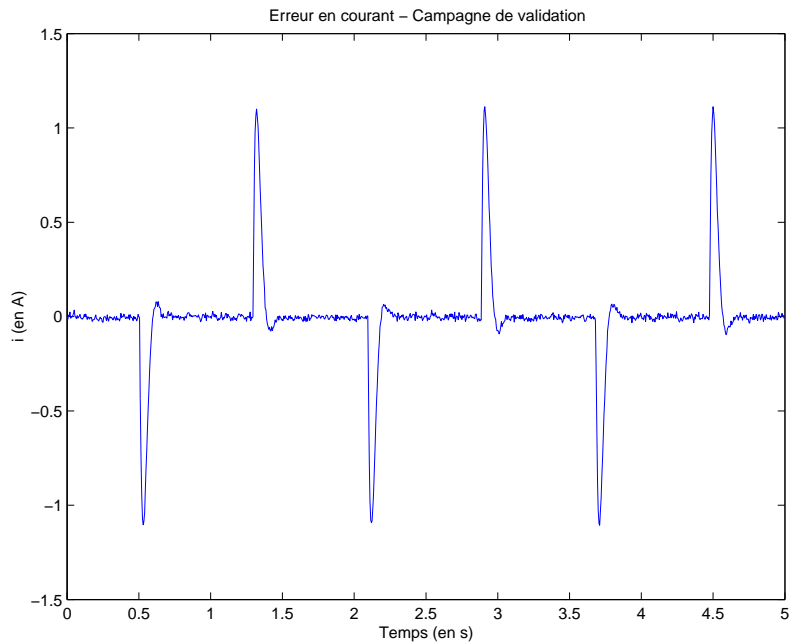

Fig. 7. Sans régularisation : évolution temporelle de l'erreur entre courants d'induit mesuré et simulé

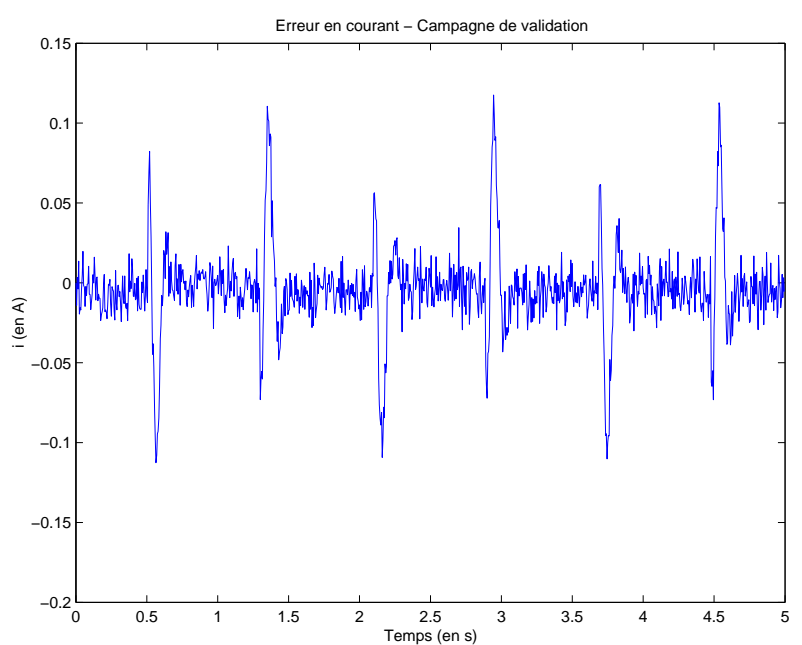

Fig. 8. Avec régularisation : évolution temporelle de l'erreur entre courants d'induit mesuré et simulé connaissances a priori de façon, ici, à pallier la propriété d'identifiabilité du modèle considéré.

L'exploitation des données expérimentales recueillies sur ce banc électro-mécanique a permis de mettre en exergue tous les résultats remarquables que peut apporter la méthodologie enseignée.

Remerciements : les auteurs remercient sincèrement leurs collègues D. Netter et F. Weinachter pour leur participation au protocole expérimental.

\section{REFERENCES}

[1] C. Defranoux. Intégration de connaissances a priori pour l'estimation de paramètres physiques de modèles à temps continu - Apport pour le diagnostic de systèmes incertains. $\mathrm{PhD}$ thesis, Thèse de Doctorat de l'Université Henri Poincaré, Nancy 1, 7 mars 2001.

[2] L. Ljung. System Identification. Theory for the user. Prentice Hall, 1987. 
[3] P. Borne, G. Dauphin-Tanguy, J-P. Richard, F. Rotella, et I. Zambettakis. Modélisation et identification des processus, Tome 1. Editions Technip, 1992.

[4] M. Abderrahim et A. R. Whittaker. Kinematic model identification of industrial manipulators. Robotics and Computer-Integrated Manufacturing, 16(1) :1-8, 2000.

[5] A. R. Da Silva et B. Schafer. Space robotics joints non-linear modeling and on-line parameters identification. Proceedings of 31st International Symposium on Robotics. 14-17 May 2000 ; Montreal, Que., Canada 2000.

[6] S. Daniel Berhe et H. Unbehauen. Physical parameter estimation of the nonlinear dynamics of a single link robotic manipulator with flexible joint using the hmf method. Proceedings of 16th American CONTROL Conference. vol.3. 4-6 June 1997; Albuquerque, NM, USA. Sponsored by : American Autom. Control Council; U.S. Nat. Member Organ. IFAC. 1997.

[7] R. Dixon et A. W. Pike. Application of condition monitoring to an electromechanical actuator : a parameter estimation based approach. Computing-8-Control-EngineeringJournal, 13(2) :71-81, 2002.

[8] Young Man Cho et Rajesh Rajamani. Identification and experimental validation of a scalable elevator vertical dynamic model. Control Engineering Practice, 9(2) :181-187, 2001.

[9] M. Ait Ahmed, S. Puren, et N. T. Maamar. Parameters identification of an induction machine using an automatic approach : simulations and experiments. Proceedings of Conference on Electrical Machines, Converters and Systems. vol.3. 14-16 Sept. 1999; Lisbon, Portugal 1999.

[10] S. Daniel Berhe et H. Unbehauen. Physical parameters estimation of the nonlinear continuous-time dynamics of a dc motor using hartley modulating functions method. Journal of the Franklin Institute, 336(3) :481-501, 1999.

[11] Guoxiu Li et Ming Fang. Physical parameters estimation of synchronous machines via blockpulse functions. Yunqiu Tang et Nai Shi, editors, Proceeding of International Conference on Electrical Machines and Applications - ICEMA'96. vol.2. 1-3 Sept. 1996; Harbin, China. Sponsored by : Natl. Nat. Sci. Found. China; Chinese Soc. Electr. Eng.; Chinese Electrochem. Soc. 1996.

[12] Li Guoxiu, Fang Ming, et Liang Darwin. Direct approach to identification of physical parameters of large generators. Proceedings of the 1996 Universities Power Engineering Conference (UPEC'96). vol.3. 18-20 Sept. 1996; Iraklio, Greece. Sponsored by : EPRI; Electr. Authority of Cyprus; General Secretariat for Res. $\mathcal{E}_{3}$
Technol., Greece; Greek Ministr. Educ.; Groupe Schneider; et al. 1996.

[13] H. Borsting, M. Knudsen, H. Rasmussen, et P. Vadstrup. Estimation of physical parameters in induction motors. M. Blanke et T. Soderstrom, editors, Proceedings of SYSID ' 94. 10th IFAC Symposium on System Identification. vol.2. 4-6 July 1994; Copenhagen, Denmark. Sponsored by : IFAC; IFORS. 1995.

[14] A. Gangopadhyay et P. H. Meckl. Extracting physical parameters from system identification of a natural gas engine. IEEE-Transactionson-Control-Systems-Technology 9(3) :425-34, 2001.

[15] M. Basset C.J. Schmitt et G.L. Gissinger. Identification of physical parameters of a passenger car. Proceedings of European Control Conference ECC'99, Karlsruhe, Germany, 31 August - 3 September, 1999, 1999.

[16] L. Le Letty et B. Nguyen. Parameter estimation in analytical models of automotive vehicles and fault diagnosis. U. Kiencke et L. Guzzella, editors, Proceedings of Advances in Automotive Control. 13-17 March 1995; Ascona, Switzerland. Sponsored by : IFAC. 1995.

[17] J.F. Bonnans, J.C. Gilbert, C. Lemaréchal, et C. Sagastizábal. Optimisation Numérique - Aspects théoriques et pratiques. Springer-Verlag, 1997.

[18] E. Walter et L. Pronzato. Identification de modèles paramétriques à partir de données expérimentales. Masson, 1994.

[19] A.N. Tikhonov et V.Y. Arsenin. Solutions of Ill-posed Problems. Winston, Washington DC, 1977.

[20] T.A. Johansen. Identification of non-linear systems using empirical data and prior knowledge - an optimization approach. Automatica, 33(3) :337-356, 1996. 